\title{
Tank monitoring: a pAMN case study
}

\author{
Steve Schneider ${ }^{1}$, Thai Son Hoang ${ }^{2}$, Ken Robinson ${ }^{2}$, and Helen Treharne ${ }^{1}$
}

\begin{abstract}
The introduction of probabilistic behaviour into the B-Method is a recent development. In addition to allowing probabilistic behaviour to be modelled, the relationship between expected values of the machine state can be expressed and verified. This paper explores the application of probabilistic B to a simple case study: tracking the volume of liquid held in a tank by measuring the flow of liquid into it. The flow can change as time progresses, and sensors are used to measure the flow with some degree of accuracy and reliability, modelled as non-deterministic and probabilistic behaviour respectively. At the specification level, the analysis is concerned with the EXPECTATION clause in the probabilistic B machine and its consistency with machine operations. At the refinement level, refinement and equivalence laws on probabilistic GSL are used to establish that a particular design of sensors delivers the required level of reliability.
\end{abstract}

Keywords: Probabilistic B, refinement, formal methods, probabilistic predicate transformers.

\section{Introduction}

This paper explores the application of probabilistic B to a simple case study: tracking the volume of liquid held in a tank by measuring the liquid flow into it. The flow can change as time progresses. Sensors with a given reliability are used to measure the flow and provide information to the system, so there is a small probability that the sensors will fail, giving an incorrect reading. The behaviour of the sensors is described using probabilistic B. We include the tank explicitly in our model so that we can describe the relationship between the actual volume of liquid it contains and our system's measurement for it. As well as probabilistic behaviour, our system exhibits nondeterministic behaviour in the reading that a failed sensor will give, and (after the first scenario we consider) in the reading that a correctly working sensor will give: any value from a particular range. Thus the case study explores the interaction between probabilistic and nondeterministic behaviour.

The case study is concerned with two stages of the development process: specification, and refinement. At the specification level we are concerned with obtaining bounds on the accuracy of the system's value for the volume of liquid in the tank, given a particular level of reliability for the combination of sensors providing the readings. This analysis will be concerned with the EXPECTATION clause in the probabilistic B machine. At the refinement level, we are concerned with establishing that a particular combination of sensors does indeed deliver the required level of reliability. This analysis will make use of refinement and equivalence laws. This paper is a full version of the work presented in [STRT05].

\footnotetext{
1 University of Surrey, UK

2 University of New South Wales, Australia
} 
The paper is structured as follows: we introduce the background approach and notation in Sections 2 and 3. Section 4 introduces the Tank case study. Section 5 introduces an initial approach to specification and refinement of a monitoring system for the Tank, introducing error margins on sensor readings of the tank. By progressively introducing more uncertainty, we investigate the significance to the specification of the possible flows of liquid into the tank, and the impact of error margins for the sensors. In Section 6, we consider a more general approach to modelling the system, in which system updates and tank updates occur separately and independently. This change in approach has a significant impact on the specification, but the refinement stage, and implementation of flow readings via the sensors, remains as it was in the previous section. The paper concludes with a discussion of what has been achieved, and some pointers to further work.

\section{The B-Method}

The B-Method [Abr96a] provides a framework for the development of provably correct systems, based on the weakest precondition semantics of the Generalised Substitution Language (GSL), and structured around the concept of Abstract Machines, described using Abstract Machine Notation (AMN). These are the fundamental components of a system description, built around the encapsulation of coherent state information, together with operations, and state invariants. Machines are associated with proof obligations, which ensure that the invariants are always preserved by the operations. Different aspects of a system description may be encapsulated within different machines, encouraging a separation of concerns. Machines may be used to describe components which will eventually be intended for code, and they may also be used to describe other system components, with which the code is to interact. In this paper we will see examples of both. The description of the Tank, given in Figure 3, is an example of a model of an external system; and the description of the VolumeTracker, given in Figure 4, is an example of a description of a software component.

Machines are described in a number of clauses, in a language known as Abstract Machine Notation. This notation is itself based on Generalised Substitution Language, a generalisation of Dijkstra's guarded command language [Dij76].

System developments are built up as combinations of machine descriptions, integrated through the B structuring mechanisms. These mechanisms are essentially of two kinds: including machines within others, and allowing read access to machines from others. They are designed to ensure that the requirements expressed through the invariants remain true, and that new requirements on the relationships between machines can be expressed and verified.

Refinement and implementation are also carried out within the framework of machines. Implementations are themselves special kinds of machine, which bear the appropriate (simulation) relationship to the corresponding abstract machine. These may themselves make use of lower-level abstract machines, leading to a layered structure of implementations. For example, the implementation of VolumeTracker given in Figure 6 makes use of some sensor abstract machines, which describe the behaviour of the individual tank sensors.

The introduction of probabilistic behaviour into the B-Method has recently been proposed [HJR $\left.{ }^{+} 03\right]$, called probabilistic $B$. This approach builds on previous work which introduces probabilistic choice into program statements, and extends the notion of weakest precondition semantics to deal with expectations [MMS96]. An expectation can be considered as the expected value of a formula or expression. Thus programs can be viewed as expectation transformers rather than predicate transformers, and their semantics gives the expectation of an expression after the program has been executed in terms of expectations prior to execution.

In addition to allowing such probabilistic behaviour into programs, probabilistic B introduces expectations on aspects of the state, in addition to the existing parts of a B machine. Thus the relationship between the expected values of several components of the machine state can be expressed and formally verified.

\section{Introducing Probability}

The introduction of probability presented in the paper remains within the context of abstract machines, and the proof obligations are augmented, to ensure that probabilistic invariants are also preserved by the probabilistic operations. 
The probabilistic generalised substitution language $p G S L$ acts over expectations rather than predicates. Expectations are bounded non-negative real-valued functions of the state space, with the exception that when dealing with miracles they can take a formal value $\infty$.

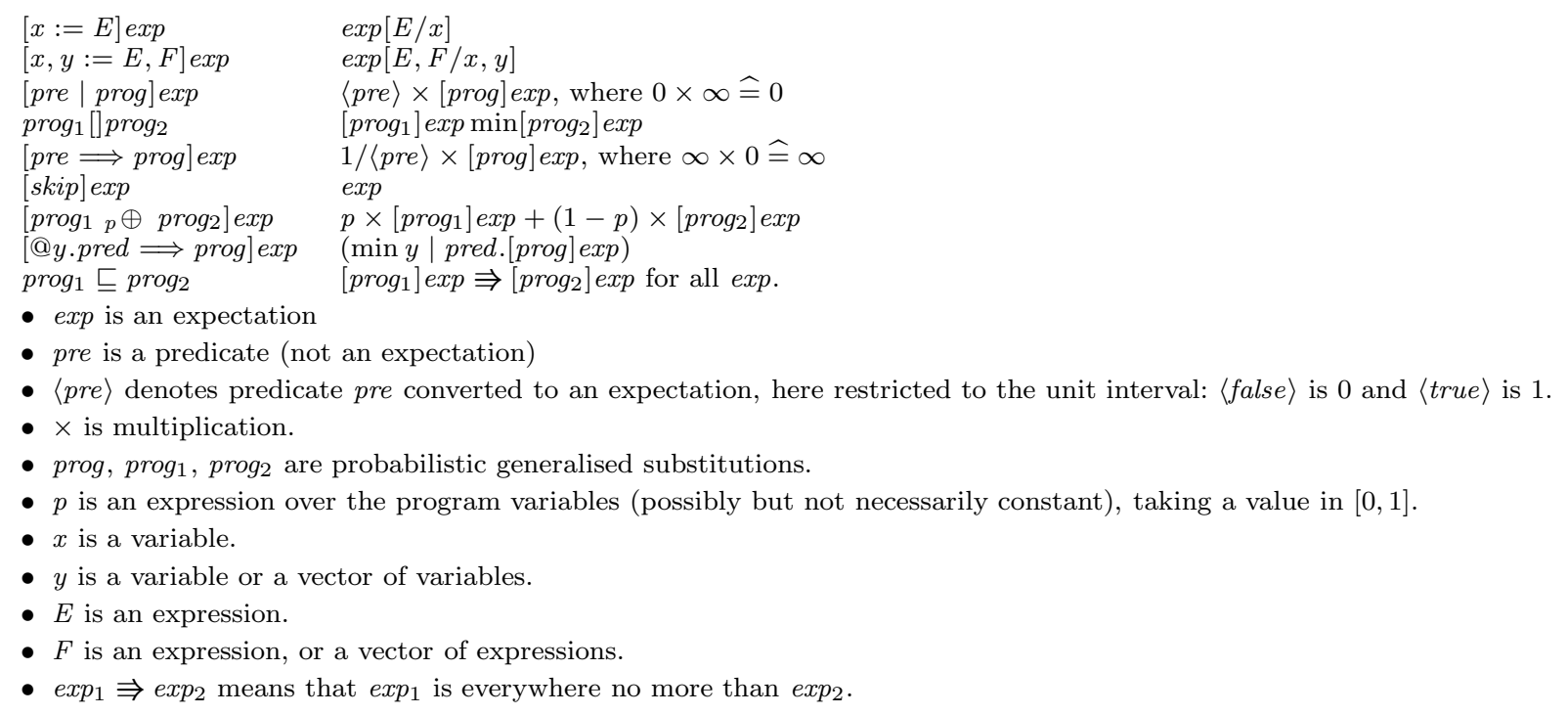

Fig. 1. $p G S L$ - the probabilistic Generalised Substitution Language [Mor98]

\subsection{Probabilistic GSL}

$p G S L$ is an extension of GSL to include a probabilistic choice statement:

$$
\operatorname{prog}_{1 p} \oplus \operatorname{prog}_{2}
$$

An execution of this choice will execute $\operatorname{prog}_{1}$ with probability $p$, and will execute $\operatorname{prog}_{2}$ with probability $1-p$. See [Mor98, MM04, MMH03] for a full introduction to pGSL.

To give a semantics to pGSL programs, we make use of expectations: bounded non-negative real-valued functions of the state space. These are generally expressed as formulas over the state variables. The weakest pre-expectation semantics for a program prog maps an expectation exp to another expectation $[\mathrm{prog}] \exp$, analogous to weakest precondition semantics. It gives the expected value for exp after prog in terms of expectations on the state before. The language and its semantics from [Mor98] is given in Figure 1.

In this paper we will use a derived operator (also given in [Abr96a]) for assigning to a variable some element chosen nondeterministically from a set $S$. We define

$$
x: \in S \widehat{=} @ y \cdot(y \in S \Longrightarrow x:=y)
$$

Thus

$$
[x: \in S] \exp =(\min x \mid x \in S . \exp )
$$

We will also use a derived operator (also given in [MM04]) for expressing a minimum probability on a choice. We define

$$
\operatorname{prog}_{1 \geqslant p} \oplus \operatorname{prog}_{2} \widehat{=} @ q \cdot(p \leqslant q \leqslant 1) \Longrightarrow \operatorname{prog}_{1} \oplus \operatorname{prog}_{2}
$$

This program chooses $\operatorname{prog}_{1}$ with a probability of at least $p$.

The operator is useful for describing systems with a minimum required reliability. If a component is required to behave correctly at least $90 \%$ of the time, then this may be described as correct $\geqslant 0.9 \oplus$ incorrect. This could be refined by a component that behaves correctly at least $95 \%$ of the time, for example. 


\subsection{Some pGSL laws}

The semantics supports a collection of algebraic laws concerning the various operators. An extended collection of laws is given in Appendix B.1.1 of [MM04], and in this paper we will make use of the following laws from that Appendix:

Law 15:

$\left(\operatorname{prog}_{1 \geqslant p} \oplus \operatorname{prog}_{2}\right) ; \operatorname{prog}_{3}=\left(\operatorname{prog}_{1} ; \operatorname{prog}_{3}\right) \geqslant p \oplus\left(\operatorname{prog}_{2} ; \operatorname{prog}_{3}\right)$

Law 20: If $\operatorname{prog}_{1}$ is a standard (i.e. not probabilistic) deterministic program, then

$\operatorname{prog}_{1} ;\left(\operatorname{prog}_{2} \geqslant p \oplus \operatorname{prog}_{3}\right)=\left(\operatorname{prog}_{1} ; \operatorname{prog}_{2}\right) \geqslant p \oplus\left(\operatorname{prog}_{1} ; \operatorname{prog}_{3}\right)$

Law 26:

$\operatorname{prog}_{1 \geqslant \overline{\bar{q}}} \oplus \operatorname{prog}_{2}=\operatorname{prog}_{1 \geqslant p} \oplus\left(\operatorname{prog}_{1 \geqslant q} \oplus \operatorname{prog}_{2}\right)$

In this law $\bar{p}=1-p$. For example, the law yields that

$x:=1 \geqslant 0.99 \oplus x:=2=x:=1 \geqslant 0.9 \oplus(x:=1 \geqslant 0.9 \oplus x:=2)$

The law is expressed in a different way in [MM04]. We express it in this way to avoid the introduction of additional notation.

We also make use of the following law, which we will call Law A:

$\operatorname{prog}_{2} \sqsubseteq \operatorname{prog}_{1} \Rightarrow \operatorname{prog}_{1 \geqslant p} \oplus \operatorname{prog}_{2}=\operatorname{prog}_{1} \oplus \operatorname{prog}_{2}$

A proof of the soundness of this law is given in Appendix B.

\subsection{Probabilistic B}

There are two aspects to the introduction of probabilistic behaviour into a $\mathrm{B}$ machine as proposed in $\left[\mathrm{HJR}^{+}\right.$03]. The first is to allow operations to be constructed using probabilistic GSL, so probabilistic choices can be made within operations. The second is to introduce an EXPECTATION clause into a B machine in order to express requirements on various expectations on the state. An EXPECTATION clause will in general contain a collection of expectation expressions. This clause plays a role for expectations analogous to the INVARIANT clause on predicates on the state. The associated proof obligations are that every operation, from any legitimate state (i.e. any state that meets the invariant), must not decrease any of the expectations.

Each expectation is of the form $e \Rightarrow V$, meaning that the expected value of $V$ is always at least the value of $e$ initially. The new proof obligations associated with each such expectation are the following:

- P1: Initialisation must establish the lower bound of the invariant. In other words, the expected value of $V$ after initialisation, $[$ Init $] V$, is at least $e$ :

$$
e \Rightarrow[\text { Init }] V
$$

- P2: Each operation must not decrease the expected value of $V$. In other words, whatever state the system is in, the expectation $V$ should not decrease when $O p$ executes:

$$
V \Rightarrow[O p] V
$$

In this paper we will use expectations of the form $V$. This is an abbreviation for $0 \Rightarrow V$. Observe that this still gives rise to a non-trivial proof obligation $P 1$, that $V$ is non-negative on initialisation.

\section{The Tank}

The system we aim to model is a tank being filled with a liquid. The liquid flows into the tank through a pipe. We wish to track the volume of liquid in the tank. This is illustrated in Figure 2. For the purposes of this example we will not be concerned with the capacity of the tank. 


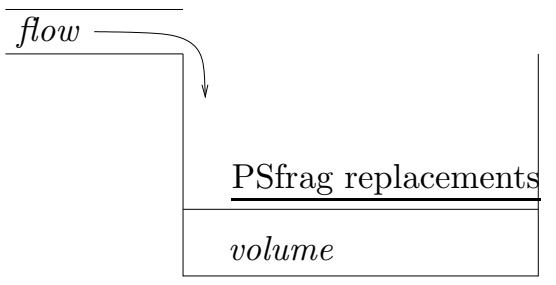

Fig. 2. The tank system

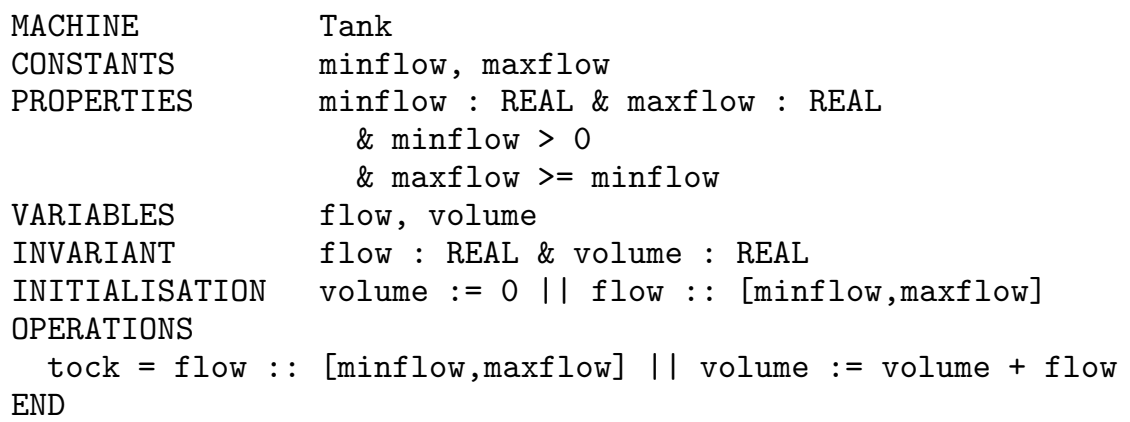

Fig. 3. The AMN description of the tank system

The tank can be modelled using the machine given in Figure $3^{3}$. This describes a model of the real tank, and will therefore be included in the specifications we will give, so that we can relate the state of the monitoring system to the real state of the tank.

Here we assume that in one time unit (as represented by tock), the volume of liquid increases by the value of flow. The value of flow can itself be any value between minflow and maxflow, and can change on every time step.

An interval of real numbers between $l$ and $h$ is denoted $[l, h]$. The interval $[x+l, x+h]$ is abbreviated $x+[l, h]$.

\section{A monitoring system}

\subsection{The first simple system}

\subsubsection{Specification}

We wish to produce a software system that tracks the volume of liquid in the tank to some level of accuracy. The system we require can be specified using the probabilistic B machine VolumeTracker 1 of Figure 4 . (The expectation makes use of values of $A$ and $B$ that will be given later.) For this first example, we take a simple approach where a single poll operation updates both the tank and the monitoring system state at the same time. Later in the paper we will consider the separation of system updates from tank updates.

Our first specification, VolumeTracker 1 , requires that a state update is perfectly accurate at least $99 \%$ of the time. Otherwise (i.e. up to $1 \%$ of the time) it can be completely arbitrary over the range of possible readings [minflow, maxflow].

The system maintains a single state variable rvolume, which contains the value the system has for the volume of liquid in the tank. Thus our specification will be concerned with the relationship between rvolume and the actual volume volume.

It is natural to have two expectations to provide a range on what the expected value for volume can be, given a particular value for the expected value of rvolume. Because rvolume and volume are increased on each

\footnotetext{
3 An explanation of the ascii form of pGSL used in Figure 3 and elsewhere in this paper is given in Appendix A.
} 


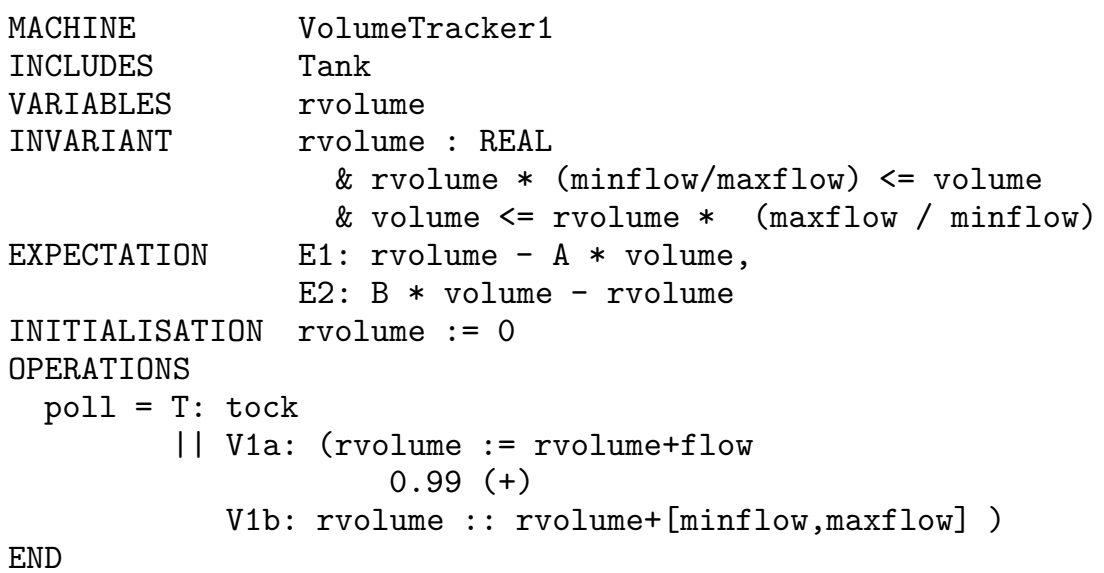

Fig. 4. The VolumeTracker 1 machine

step with some value from a fixed range of possible values, we consider expectations as linear combinations of rvolume and volume. Thus they would be of the form:

E1: rvolume $-A \times$ volume

E2: $B \times$ volume - rvolume

These must both be non-negative, so we can deduce for the expected values that

$$
\text { rvolume } B \leqslant \text { volume } \leqslant \text { rvolume } / A
$$

Thus given an expected value for rvolume we have a range for the expected value of volume. The required degree of accuracy as given by $A$ and $B$ will naturally emerge as part of the specification.

Since both $E 1$ and $E 2$ must be greater than 0 , and non-decreasing on every occurrence of poll, we obtain some constraints on the possibilities for $A$ and $B$.

Observe that any absolute restrictions on the relationship between volume and rvolume will appear in the invariant. In particular, the lower and upper bounds on volume for any given value of rvolume are given by the following inequalities:

$$
\text { rvolume } \times(\text { minflow } / \text { maxflow }) \leqslant \text { volume } \leqslant \text { rvolume } \times(\text { maxflow } / \text { minflow })
$$

This will always be true, so it is included in the invariant. However, it does not provide a very tight relationship between volume and rvolume.

\subsubsection{Deriving $A$ and $B$}

For VolumeTracker 1 to meet its proof obligations, we require that the expectations will never decrease on any call of the operation poll, from any state.

We can carry out some calculations to derive conditions for $A$ and $B$ to achieve this. We require that $E 1 \Rightarrow[p o l l] E 1$ and $E 2 \Rightarrow[$ poll $] E 2$. Thus we require that for any flow, volume, and rvolume, we must have that $([$ poll $] E 1)-E 1 \geqslant 0$ and $([$ poll $] E 2)-E 2 \geqslant 0$.

We calculate the requirement on $A$ from the requirement on $E 1$ :

$$
\begin{aligned}
([\text { poll }] E 1)-E 1= & \left(\left[T \|\left(V 1 a{ }_{0.99} \oplus V 1 b\right)\right] E 1\right)-E 1 \\
= & ([T \| V 1 a) 0.99 \oplus(T \| V 1 b)] E 1)-E 1 \\
= & (0.99 \times[T \| V 1 a] E 1+0.01 \times[T \| V 1 b] E 1)-E 1 \\
= & (0.99 \times(\text { rvolume }+ \text { flow }-A \times(\text { volume }+ \text { flow })) \\
& \quad+0.01 \times(\text { rvolume }+ \text { minflow }-A \times(\text { volume }+ \text { flow }))) \\
& \quad-(\text { rvolume }-A \times \text { volume })
\end{aligned}
$$




$$
\begin{aligned}
& =0.99 \times(\text { flow }-A \times \text { flow })+0.01 \times(\text { minflow }-A \times \text { flow }) \\
& =(0.99-A) \times \text { flow }+0.01 \times \text { minflow }
\end{aligned}
$$

Since this must be non-negative everywhere (i.e. for all possible values of flow), we obtain that

$$
A \leqslant 0.99+0.01 \times(\text { minflow } / \text { flow })
$$

for any value of flow. The bound takes its minimal value when flow is maxflow, so we obtain that

$$
A \leqslant 0.99+0.01 \times(\text { minflow } / \text { maxflow })
$$

Thus the closer to 1 the ratio between minflow and maxflow, the closer $A$ can be to 1 and the more accurate the upper bound on the expected value for volume for any given expectation on rvolume. However, note that $A$ can always be at least 0.99 .

For $B$ we perform the following calculation:

$$
\begin{aligned}
([\text { poll }] E 2)-E 2= & \left(\left[T \|\left(V 1 a_{0.99} \oplus V 1 b\right)\right] E 2\right)-E 2 \\
= & \left(\left[(T \| V 1 a)_{0.99} \oplus(T \| V 1 b)\right] E 2\right)-E 2 \\
= & (0.99 \times[T \| V 1 a] E 2+0.01 \times[T \| V 1 b] E 2)-E 2 \\
= & (0.99 \times(B \times(\text { volume }+ \text { flow })-(\text { rvolume }+ \text { flow })) \\
& \quad+0.01 \times(B \times(\text { volume }+ \text { flow })-(\text { rvolume }+ \text { maxflow }))) \\
& \quad-(B \times \text { volume }- \text { rvolume }) \\
= & 0.99 \times(B \times \text { flow }- \text { flow })+0.01 \times(B \times \text { flow }- \text { maxflow }) \\
= & B \times \text { flow }-0.99 \times \text { flow }-0.01 \times \text { maxflow }
\end{aligned}
$$

We require that this is non-negative for any value of flow. Thus $B \geqslant 0.99+0.01 \times($ maxflow/flow) for any value of flow. The largest value for the expression (i.e. the largest lower bound for $B$ ) is given when flow $=$ minflow, and we obtain

$$
B \geqslant 0.99+0.01 \times(\text { maxflow } / \text { minflow })
$$

Observe lines (2) and 3) concerning the evaluation of $[T \| V 1 b]$ with respect to an expectation. Since $V 1 b$ is nondeterministic in the assignment to rvolume, the minimum expectation over all possible assignments to rvolume must be taken. In $E 1$, rvolume is positive, so the smallest possible value of rvolume is used in the calculation of the pre-expectation of $E 1$. In $E 2$ rvolume is negative so the largest possible value of rvolume is used in the calculation of the pre-expectation of $E 2$. This means that however the nondeterminism is later resolved, the expectation will be at least the value calculated. Expectations should always be non-decreasing, so demonic nondeterminism always considers the worst case with respect to increases.

\subsubsection{Example}

As an illustration, we shall consider some concrete numbers: if minflow $=100$ and maxflow $=400$, then we obtain $A \leqslant 397 / 400=0.9925$ and $B \geqslant 103 / 100=1.03$. Thus we deduce from inequality (1) above that

$$
(100 / 103) \times \text { rvolume } \leqslant \text { volume } \leqslant \text { rvolume } \times(400 / 397)
$$

This implies for example that

$$
0.97 \times \text { rvolume } \leqslant \text { volume } \leqslant 1.03 \times \text { rvolume }
$$

so if we have a requirement for $97 \%$ accuracy, this will be met.

However, if our requirement is for $99 \%$ accuracy, this will not be met. The description cannot ensure that $0.99 \times$ rvolume $\leqslant$ volume. This is because an incorrect reading, that could occur with probability 0.01 , could be wrong by a factor of 4 , leading to a large increase of rvolume over the real value of volume. The level of accuracy is concerned not only with the probability of correct readings, but also with the amount by which a flawed reading could be out.

To ensure $99 \%$ accuracy we would either have to reduce the ratio between minflow and maxflow (so bad readings cannot be so wildly out), or decrease the probability of a bad reading. Observe that these alterations are concerned only with the specification machine. This machine gives the probability of an accurate reading that is required for ensuring the expectations. 


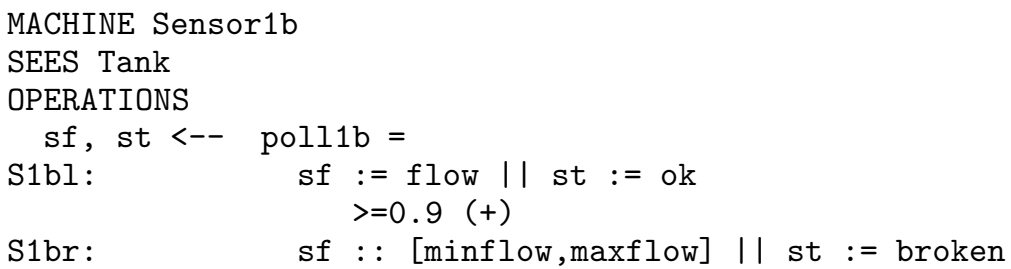

END

Fig. 5. A Sensor machine

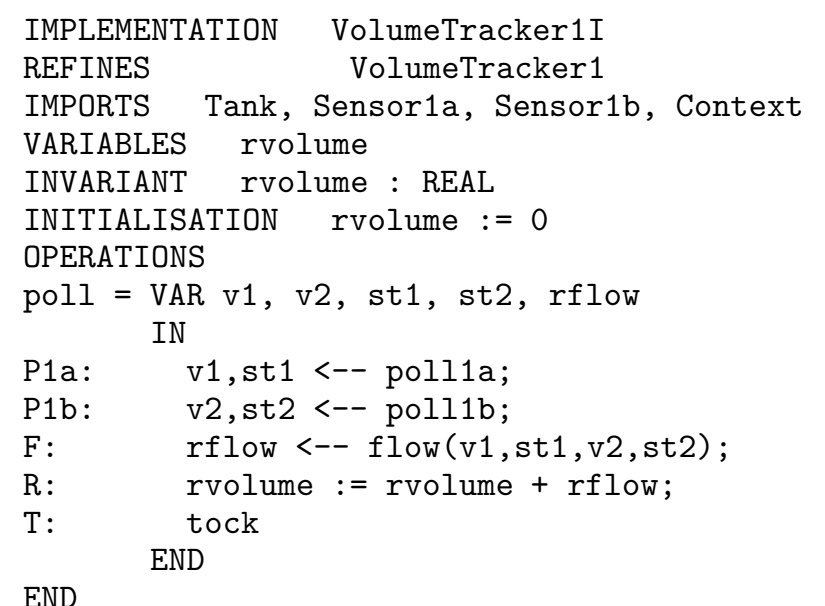

Fig. 6. The implementation VolumeTracker $1 I$

\subsubsection{Implementation}

Our first implementation of VolumeTracker 1 will make use of two sensors, which provide readings for the flow, and also give diagnostic information stating whether they are broken or not. We will firstly consider sensors which can fail on any particular reading independently of any other reading. We will consider sensors which have a reliability of at least $90 \%$. We will need to make use of two of these, Sensor $1 a$ and Sensor $1 b$ to give readings to $99 \%$ accuracy. Sensor $1 b$ is given in Figure 5 , and Sensor $1 a$ is entirely similar.

We propose an implementation VolumeTracker $1 I$ of VolumeTracker 1 which uses two sensors in order to obtain a more reliable reading of the flow. This is given in Figure 6, and makes use of the Context machine of Figure 7.

Observe that the implementation contains its own variable rvolume. To avoid complicating this example with imported state, we relax the normal restriction that implementation machines cannot have their own state.

We need to prove that the poll operation in the implementation is a refinement of the poll operation in the specification. This can be done by manipulating the probabilistic choices using the laws of [MM04] given in Section 3.2.

The poll operation in VolumeTracker $1 I$ of Figure 6 is of the form $P 1 a ; P 1 b ; F ; R ; T$, where the variables $v 1, v 2$, st 1 , st 2 , rflow are all local. We show that this operation is equivalent to poll given in the specification machine VolumeTracker 1 , as follows:

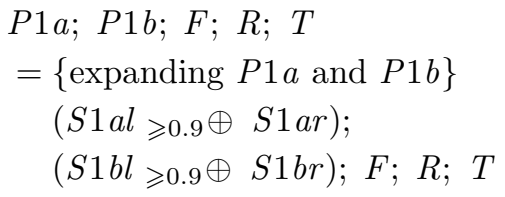




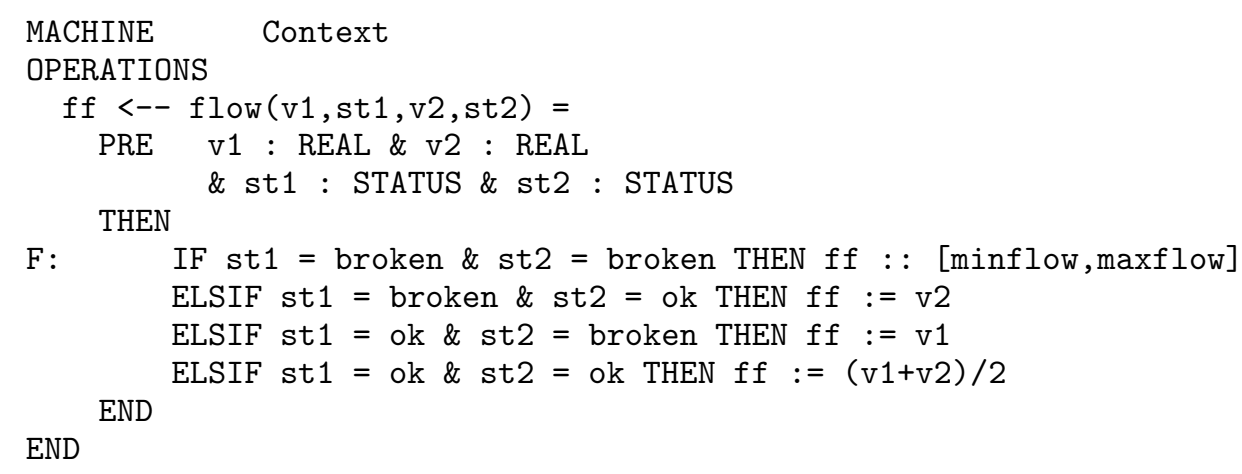

Fig. 7. The AMN description of flow calculation

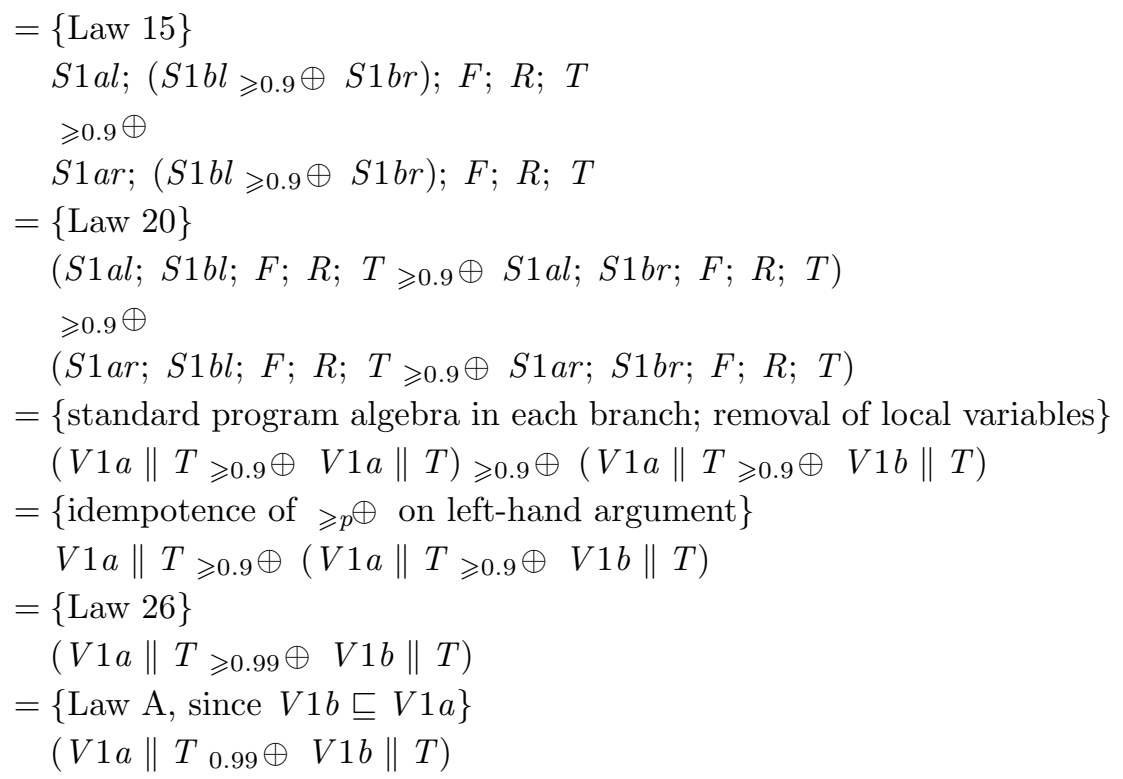

Thus we arrive at the operation poll given in the specification machine VolumeTracker 1 . This demonstrates that VolumeTracker $1 I$ indeed provides an implementation of VolumeTracker 1.

\subsubsection{Summary}

This first example has illustrated several points:

- The expected value of the machine expectation expression should be non-decreasing on every occurrence of the operation.

- However, the actual value of the machine expectation expression can decrease on some operation calls (provided its expected value does not).

- Expectations can be used to express a relationship between the expected values of state variables, in our case providing a range for the expected value of volume in terms of the expected value of rvolume. This is checked as part of machine consistency, and is independent of any particular implementation.

- The accuracy of the approximation rvolume to the tank value volume depends not only on the probability of an incorrect reading, but also on the ratio between minflow and maxflow, since this affects the maximum possible error in rvolume.

- Probabilistic operations can be implemented using combinations of probabilistic components (sensors) in the way we would expect. Such implementations need only be checked for refinement against the machine 


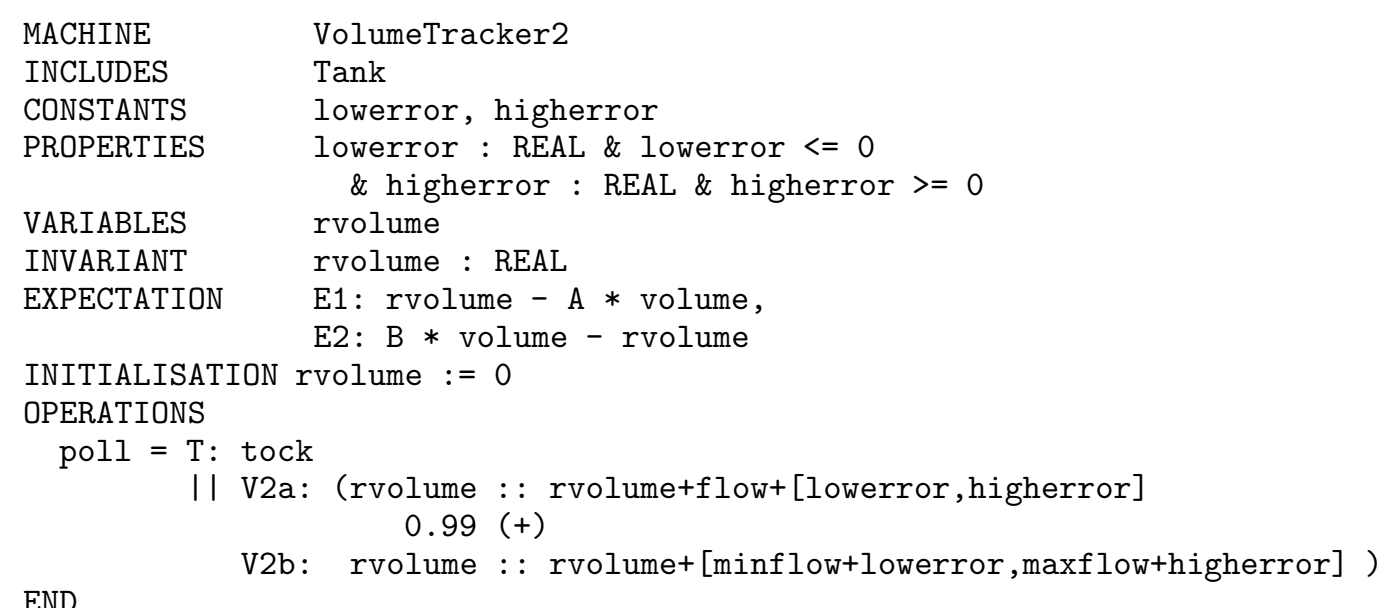

Fig. 8. The AMN description of the second monitoring system

descriptions of the operations. The machine consistency checks ensure that the machine operations provide the overall requirements on the expectations.

\subsection{Introducing error margins}

\subsubsection{Specification}

In the previous example, we did not allow for any error on reading flow. We now allow for a margin of error in readings of flow. Specifically, the error can be any value in the range [lowerror, higherror]. Typically the possibility of no error at all should be within the range, so lowerror will be negative and higherror will be positive. The revised machine is given in Figure 8.

The calculation of appropriate $A$ and $B$ follows the same pattern as shown previously in Section 5.1.2, and is given in Appendix C. Now two sources of nondeterminism must be taken into account: the reading of the sensors in $V 2 a$ (which can be most pessimistic with regard to $E 1$ when flow is low) and the arbitrary reading in $V 2 b$ (which can be most pessimistic for $E 1$ when flow is high). This combination of considerations (recall lowerror is negative, so $A \leqslant 1$ ) means that $A$ is bounded above by both of the following values:

$$
1+(\text { lowerror/minflow })
$$

and

$$
0.99+(\text { lowerror } / \text { maxflow })+0.01 \times(\text { minflow } / \text { maxflow })
$$

For example, if minflow $=100$, maxflow $=400$, and lowerror $=-10$, then the first value is lower, and we obtain $A=0.9$. On the other hand, if lowerror $=-0.1$, then the second value is lower and we obtain $A=0.9915$. In the first case the possible error in any reading of the flow is $10 \%$ of minflow, so the worst case occurs when the flow is minflow and minflow + lowerror is added to rvolume: the resulting rvolume could be $10 \%$ out. On the other hand, in the second case the error in the flow can be at most $0.1 \%$, so the error that can be introduced by $V 2 b$ ( $1 \%$ of the time) dominates, and the worst case occurs when the flow is maxflow and rvolume is only incremented by lowerror + minflow.

Similar considerations for the expectation $E 2$ yield that the value obtained for $B$ is the maximum of the following two values, the first for the case where flow = maxflow and the second when flow $=$ minflow.

$$
1+(\text { higherror/maxflow) }
$$

and

$$
0.99+(\text { higherror } / \text { minflow })+0.01 \times(\operatorname{maxflow} / \text { minflow })
$$

In this case, the second value will always be higher, and hence will give the appropriate value for $B$, since 


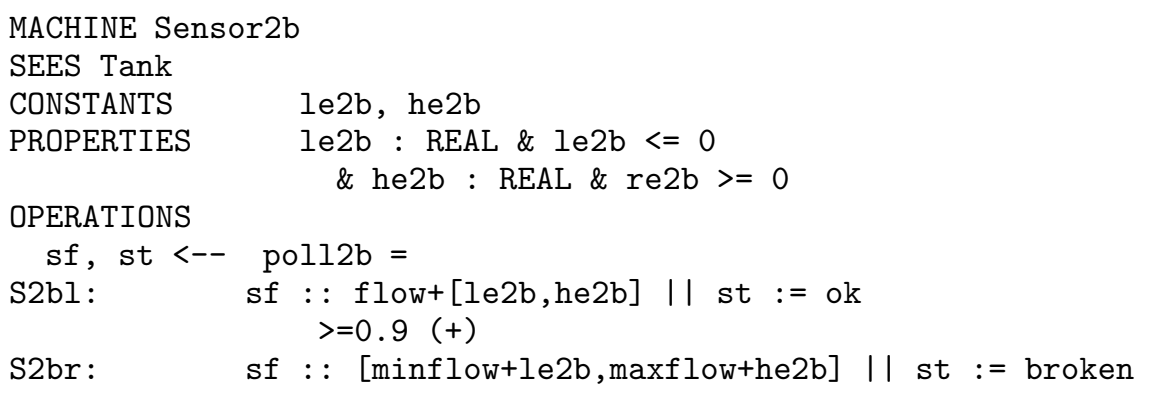

END

Fig. 9. The machine Sensor $2 b$

maxflow/minflow $\geqslant 1$, and higherror/minflow $\geqslant$ higherror/maxflow. This informs us that the worst case always occurs with a flow of minflow, and an incorrect reading of maxflow + higherror. This is worse than the worst outcome that can be obtained with a flow of maxflow, as far as ensuring that $E 2$ does not decrease is concerned.

\subsubsection{Implementation: sensors}

The error is likely to have been included in the specification because the sensors introduce some error. We can include these errors within the sensor descriptions, resulting in a new version of the sensor description. For example, in Sensor $2 b$ we will take the error range to be $[l e 2 b, h e 2 b]$. The resulting sensor is given in Figure 9.

The implementation VolumeTracker $2 I$ will be the same as VolumeTracker $1 I$, except that it now imports Sensor $2 a$ (with error range [le $2 a, h e 2 a]$ ) and Sensor $2 b$, instead of the original sensors. It is given for reference in Figure 17 of Appendix D.

Observe that in this scenario two sensors working correctly might not agree on their readings. In this case the context machine specifies that the average of the two readings should be taken.

The machine VolumeTracker $2 I$ provides an implementation of poll, provided the following hold: that $[l e 2 a, h e 2 a] \subseteq[$ lowerror, higherror $]$ and $[$ le $2 b, h e 2 b] \subseteq[$ lowerror, higherror $]$. In other words, that the error ranges for each sensor are within those given in VolumeTracker 2 for the overall combination. The proof of this is given in Appendix D.

\subsubsection{Summary}

This second example illustrates several points:

- We can specify error ranges for readings of flow.

- Such ranges have an impact on the expectations that will be non-decreasing on operations: the nondeterminism in the state updates means that the relationship between rvolume and volume will be weaker.

- The particular relationships that can be guaranteed between volume and rvolume depend on the error ranges of readings and also on the the ratio of maxflow to minflow. Each of these dominates in some cases.

- The flow readings can be implemented by sensors whose errors are within the specified range.

\subsection{Removing sensor diagnostics}

We now consider the situation where the sensors do not provide explicit status information. In this case the only way faulty readings can be identified is by comparison with other readings.

In this example we will work from the sensors to the specification: we will derive the specification that the combination of sensors delivers. 


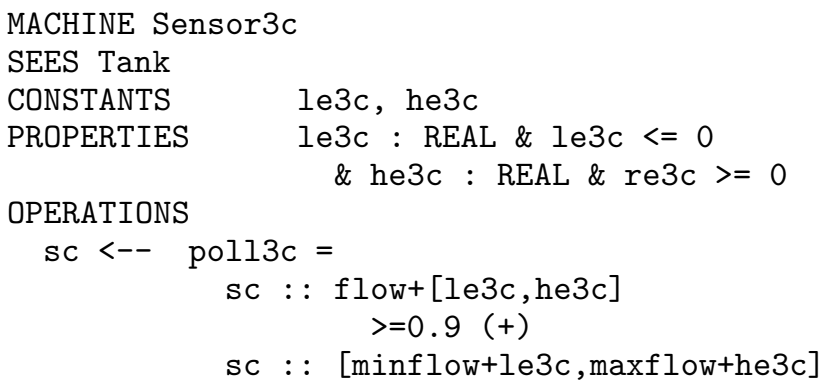

END

Fig. 10. A sensor without diagnostics

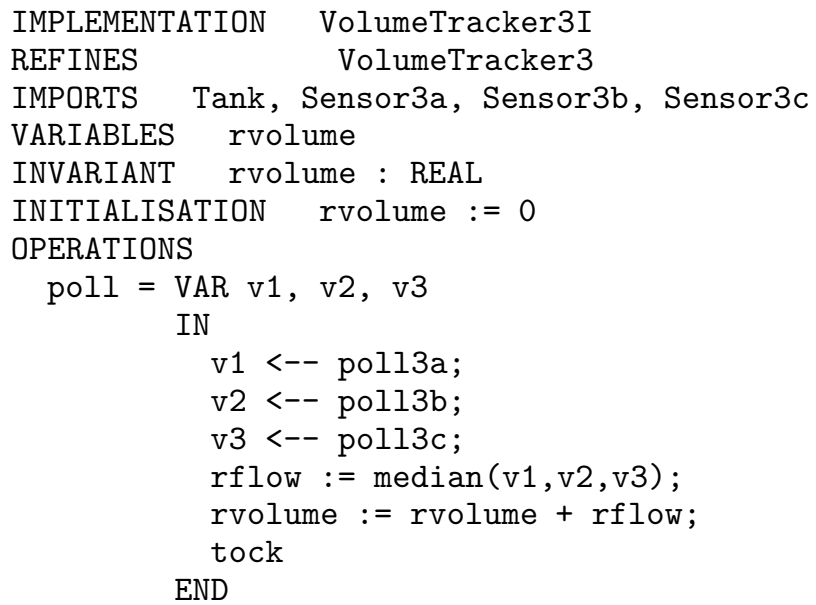

END

Fig. 11. The implementation VolumeTracker $3 I$

\subsubsection{Implementation: sensor}

A sensor without diagnostic information about its status is given in Figure 10. It provides only a flow reading, without any information about its state.

To be tolerant to one faulty reading, we need three sensors: Sensor $3 a$, Sensor $3 b$, and Sensor $3 c$. By taking the median value of the three readings we obtain an accurate reading, provided no more than one of them goes wrong. This suggests the implementation given in Figure 11. We still assume a 90\% reliability on the reading of any individual sensor.

\subsubsection{Specification}

In fact here VolumeTracker $3 I$ is a refinement of the specification VolumeTracker 3 given in Figure 12, provided all of the sensor errors are within the error given in VolumeTracker3, e.g. [le3, he3] $\subseteq$ [lowerror, higherror].

For VolumeTracker 3, carrying out the standard calculations on preservation of $E 1$, we find that the best (highest) value we can obtain for $A$, which enables the expectation $E 1$ to be preserved, is the minimum of

$$
1+(\text { lowerror/minflow })
$$

and

$$
0.972+0.028 \times(\text { minflow } / \text { maxflow })+\text { lowerror } / \text { maxflow }
$$




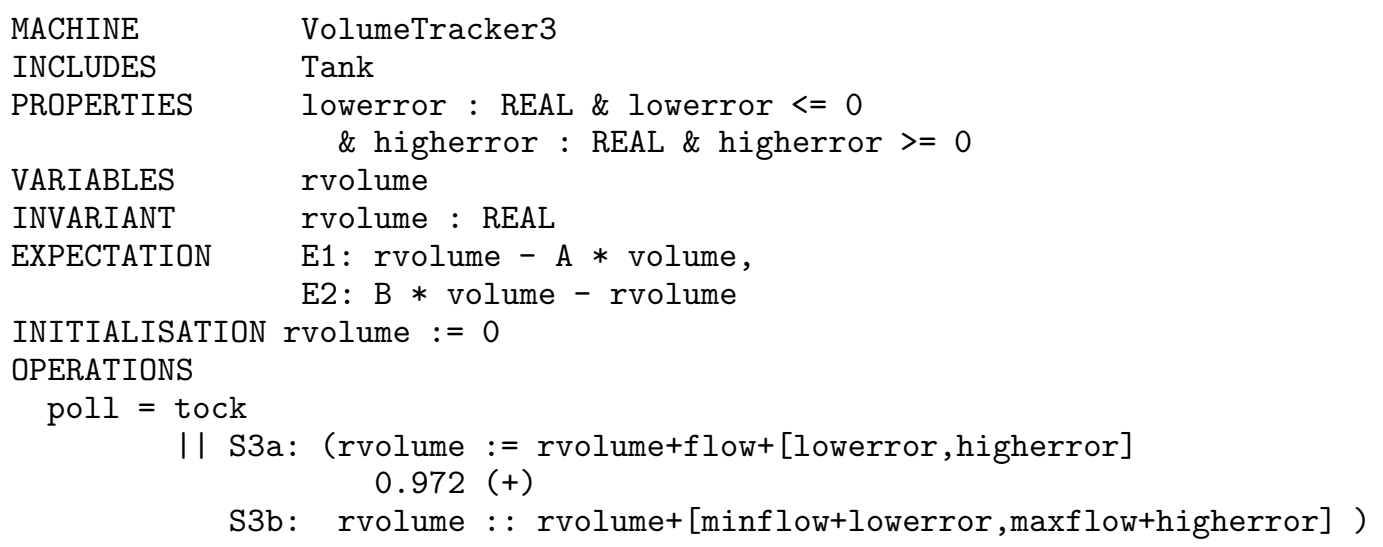

END

Fig. 12. The third monitoring system specification

Similarly, the best (lowest) value we can obtain for $B$ is the maximum of

$$
1+(\text { higherror/maxflow) }
$$

and

$$
0.972+0.028 \times(\text { maxflow } / \text { minflow })+(\text { higherror } / \text { minflow })
$$

The second of these will always be the maximum, since maxflow $\geqslant$ minflow. The situation is similar to the previous example considered in Section 5.2.2, but with a probability of an incorrect reading now at 0.028 rather than 0.01 . Thus the expectations on the relationship between rvolume and volume are correspondingly weaker, since more weighting is given to the ratio between maxflow and minflow.

For example, consider the situation where we have maxflow $=400$, minflow $=100$, higherror $=1$, lowerror $=-1$.

Since the expectation $E 1=$ rvolume $-A \times$ volume must not decrease, whatever the value of flow, we have two extremes to consider:

- If flow = minflow, then volume is incremented by minflow, and the least that rvolume can be incremented by is minflow + lowerror. Thus in this case we obtain a possible value of $A=0.99$.

- If flow = maxflow, then volume is increased by maxflow, and the least that rvolume can be incremented by is minflow + lowerror if at least two sensors go wrong (which can happen with probability 0.028), otherwise maxflow +lowerror. Thus the most pessimistic expectation gives a possible value of $A=0.9765$. Here the ratio between maxflow and minflow is more significant than the ratio between minflow and lowerror in contributing to the amount by which rvolume can be down, and we obtain a value of 0.9765 for $A$.

We also require that the expectation $E 2=$ volume $-B \times$ rvolume must not decrease. Here we are concerned with the proportion by which volume can exceed rvolume, and the worst case always occurs when flow $=$ minflow. In this case, the reading might at worst be maxflow + higherror (with probability 0.028) and minflow + higherror otherwise. This yields a value for $B$ of at least 1.094 if the expectation of $E 2$ is not to decrease. This is a margin of error of $9.4 \%$.

\subsubsection{Summary}

This version of the tank monitoring system has considered a version of sensor which does not provide feedback on its status. Thus a sensor's incorrect reading can only be discovered by comparing it with other sensors. We considered an implementation which uses three sensors in such a way that if at most one has failed then an accurate reading is obtained. We found that if each sensor has at least $90 \%$ reliability, then the combination has at least $97.2 \%$ reliability in terms of providing an accurate reading. This allowed us to construct the specification that was guaranteed by the implementation. This in turn enables the relationship between the expected values of volume and rvolume to be established. 


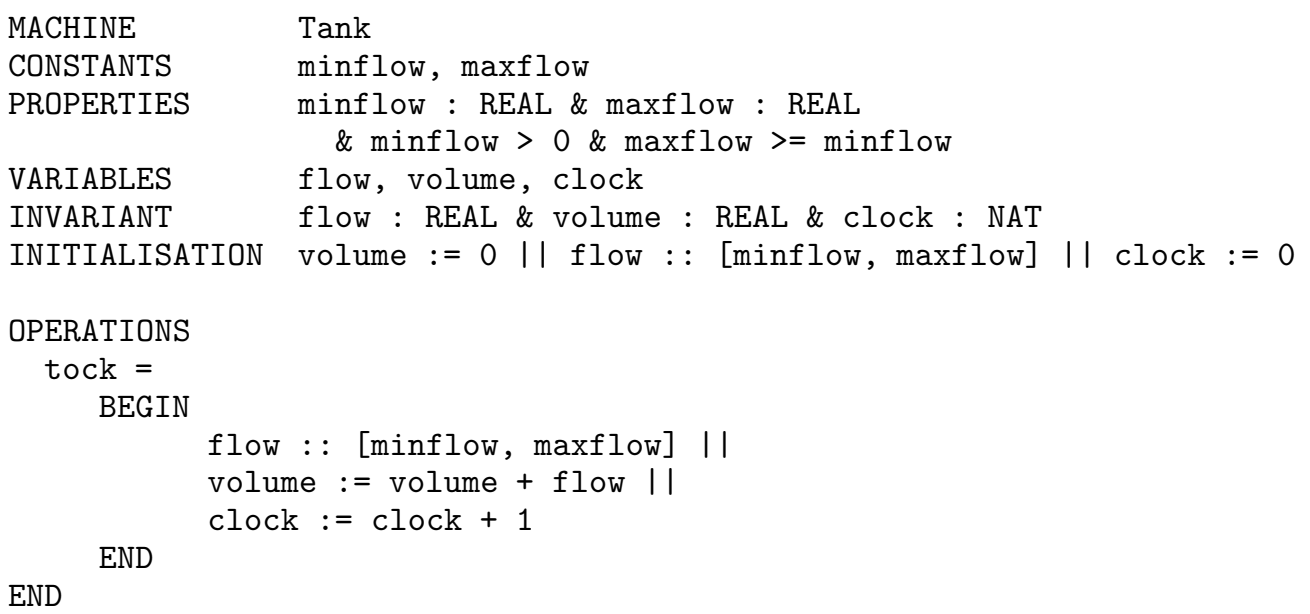

Fig. 13. The new model of the tank, tracking the number of updates

\section{Separating system updates and tank updates}

It could be useful to separate the model of the tank from the model of the system, and not refer to tank updates in the poll operation at all. Consequently, we could keep this abstract tank update operation throughout the refinement, and then discard it once we have all the implementation. This is a small change from conventional B where we would expect to use all the operations of a machine's implementation, but it is appropriate in modelling embedded systems [DT97], and a similar approach also occurs in Event-B [Abr96b, Abr03]. Here, we want to keep only the operations which model the actual software functionality, and discard the model of the environment once it is no longer required. Normally the environment model is needed only at the abstract level in order to specify a safety property between the approximated value rvolume of the volume in the tank and the real value volume, as we have seen in the expectations of the VolumeTracker machines previously.

To achieve this separation, we revise the use of tock and poll. The operation tock advances flow and volume as before, and also advances time. This operation represents what is happening in the physical tank. The operation poll increases rvolume in line with the flow as previously, but does not now call tock as part of its operation. Instead, it brings the value of rvolume up to date by noting the flow and the amount of time that has elapsed since the last poll. The poll operation is the one we would want to implement.

Now it is possible (indeed inevitable) that there will be some states of the system where volume and rvolume do not match, because tock and poll are out of step. Furthermore, any expectation of the form rvolume $-A \times$ volume must decrease on tock, since that increases volume but does not change rvolume. Similarly, an expectation of the form $B \times$ volume - rvolume must decrease on poll, since that increases rvolume while leaving volume unchanged. Thus we require a way of dealing with the separation of tock from poll.

There are in fact a variety of approaches we could take to dealing with this in the specification. In this section we will explore the introduction of auxiliary variable clock to track the number of times the tock has been called, and $a a$ to track the system's approximation to clock, updated whenever poll is called. We will include this information in the expectations.

\subsection{A first attempt}

The description of the tank model is given in Figure 13. It incorporates a new variable clock to track the number of times tock has been called. Observe that flow can change on each occurrence of this operation.

The new monitoring system is given in Figure 14. This includes the model of the tank, and introduces its own counter aa for tracking the number of calls to poll.

The expectations $E 1$ and $E 2$ that would be appropriate to include will need to take into account the difference between $a a$ and clock. The general form of such expectations will be as follows: 


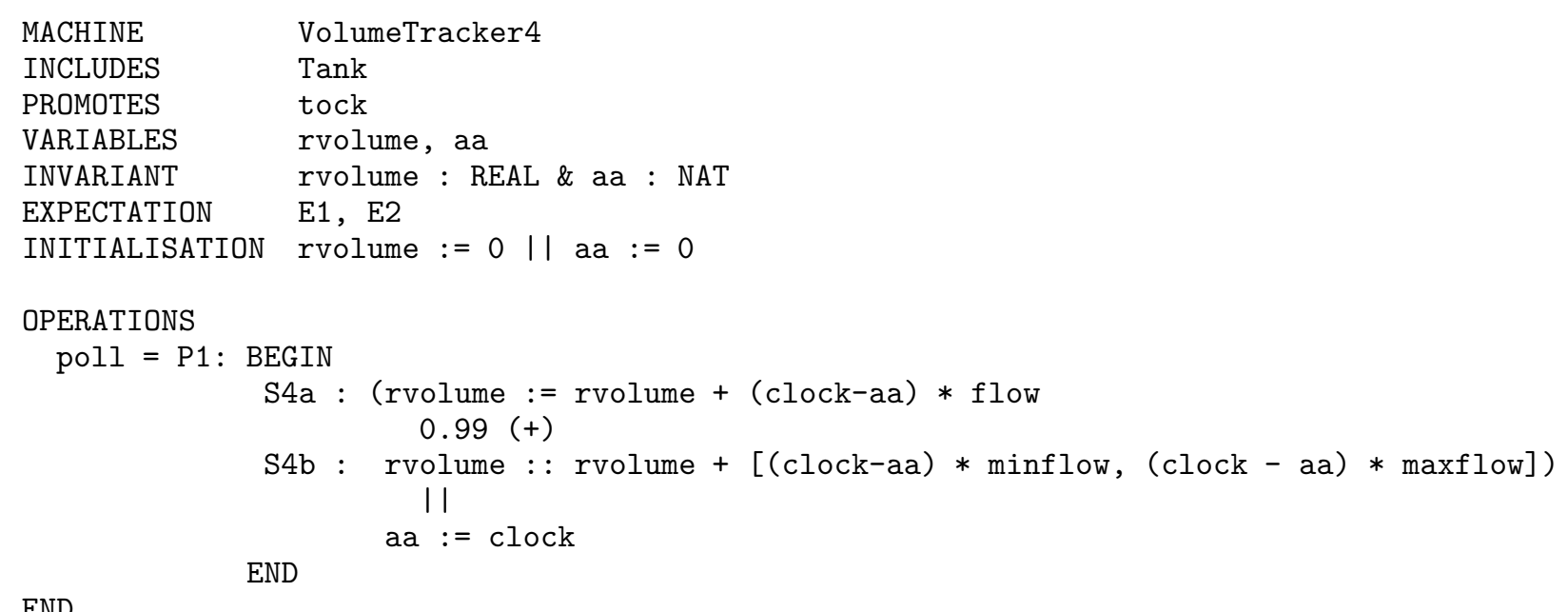

Fig. 14. A tank monitoring system separating system from tank updates

- E1: rvolume $-A \times$ volume $-(a a-$ clock $) \times A^{\prime}$

- E2: $B \times$ volume - rvolume $-($ clock $-a a) \times B^{\prime}$

These expectations must be non-decreasing on every operation. Thus they must both be preserved by both poll and tock. In the case of $E 1$, poll will increase rvolume and $a a$, so the increase in $a a$ can be used to offset the increase in rvolume, which in this operation is not matched by a corresponding increase in volume. Similarly, tock will increase volume and clock. Thus the decrease in $(a a-$ clock) will be used to offset the decrease in rvolume $-A \times$ volume, so that the overall expectation does not decrease. The appropriate values for $A$ and $A^{\prime}$ can be calculated by using the inequalities $[p o l l] E 1 \Rightarrow E 1$ and $[$ tock $\left.] E 1\right] \Rightarrow E 1$.

A similar form of reasoning applies to $E 2$, and we obtain the following instantiations:

- E1: rvolume $-($ minflow/maxflow $) \times$ volume $-($ aa - clock $) \times$ minflow

- E2: $($ maxflow $/$ minflow $) \times$ volume - rvolume $-($ clock - aa $) \times$ maxflow

These expectations provide very weak bounds. The difficulty that this calculation has highlighted is that poll and tock will in general be updating rvolume and volume with different values of flow. In the most extreme case, tock could perform a number of updates with flow = maxflow, and then poll could perform an update with flow = minflow. In general, if the machines become more out of step (which is certainly allowed within the specification), then volume and rvolume might be incremented with different values of flow, and so could become quite different. Since flow is updated nondeterministically on occurrences of tock, we must consider the worst case possibility, and this is so bad that it completely overshadows any probabilistic behaviour that we might hope to describe in the expectation.

\subsubsection{Summary}

In this example we have seen how the updates to the monitoring system and to the tank can be separated. This separation introduces the possibility that the real value volume and the system value rvolume can diverge quite considerably, for two reasons: firstly, tock and poll might not occur together in general, so one might occur much more than the other; and secondly, tock and poll in general will read different values of flow, and so the updates they effect can be different, even if they are reasonably closely in step.

The expectations must be non-decreasing for both operations however the nondeterminism is resolved. The separation of tock and poll means that the relationship between the expected values of volume and rvolume is weakened.

Note that the implementation of the poll operation in terms of sensors will be the same as it was previously (except that tock will not now be included). A reading of the flow by means of two or three sensors as in the Section 5 will provide a suitable implementation of the operation exactly as it did before. 


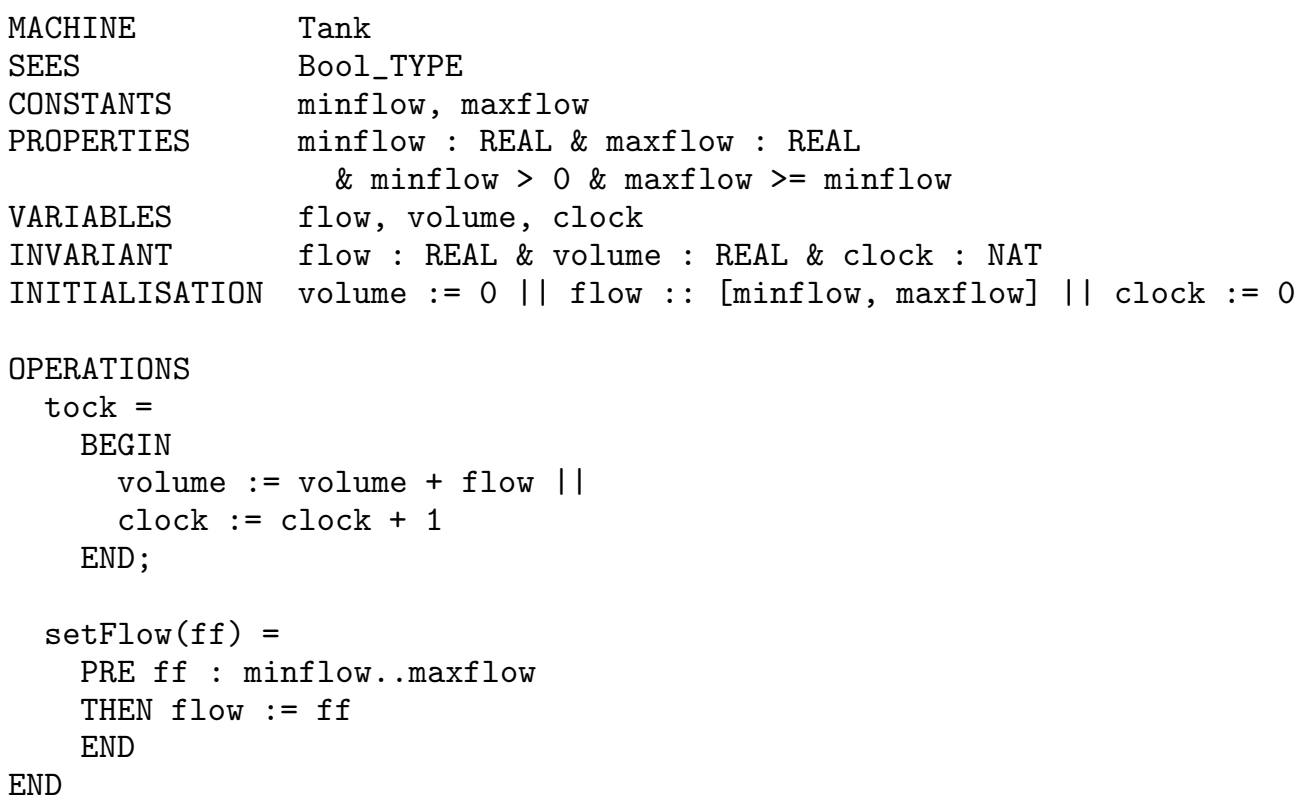

Fig. 15. A tank monitoring system separating system from tank updates

\subsection{Restricting flow changes}

The first approach to separating tock from poll yielded a very weak relationship between the system and the real values for the volume of the liquid in the tank. However, in practice we might have certain assumptions about the way these operations might be called. For example, we might expect poll to be called sufficiently frequently that flow does not change between occurrences of tock and of poll. We can incorporate this into the model by introducing an explicit operation setFlow which is the only operation that changes the flow; and we can include an assumption (by means of a precondition) that this only occurs when tock and poll are in step. This builds our environmental assumptions, that calls of poll will be sufficiently effective to keep the system up to date, into the model. In fact we still allow rvolume and volume to become out of step, but in a controlled way, as we will see below. The updated version of the tank is given in Figure 15.

One way to incorporate this is to call setFlow from within the monitoring system, under a precondition so that the flow can be changed only when rvolume and volume are in step. Of course this precondition involves both the software and the tank system, and incorporates a modelling assumption.

If the flow can be changed while the system is out of step, then a much weaker expectation would result, in the extreme case corresponding to the expectations derived in Section 6.1 - a rather weaker relationship between rvolume and volume. Since we are concerned to exclude that, we will include the precondition in setNewFlow. The resulting machine is given in Figure 16. The expectations in VolumeTracker4a must be non-decreasing under all three operations. We obtain the following expectations:

- E1: (rvolume $-(0.99+0.01 \times($ minflow $/$ maxflow $)) \times$ volume $)$

$$
+(\text { clock }-a a) \times((0.99+0.01 \times(\text { minflow } / \text { maxflow })) \times \text { flow })
$$

- E2: $(0.99+0.01 \times($ maxflow $/$ minflow $) \times$ volume - rvolume $)$

$$
+(\text { aa }- \text { clock }) \times((0.99+0.01 \times(\text { maxflow } / \text { minflow })) \times \text { flow })
$$

Observe that setNewFlow does not change the expectations $E 1$ and $E 2$, because its precondition states that clock $=a a$, so the part of the expectation dependent on flow evaluates to 0. Observe also that the extent by which rvolume and volume are out of step is accounted for by a multiple of flow, which has been constant since the last time volume and rvolume were properly aligned. 


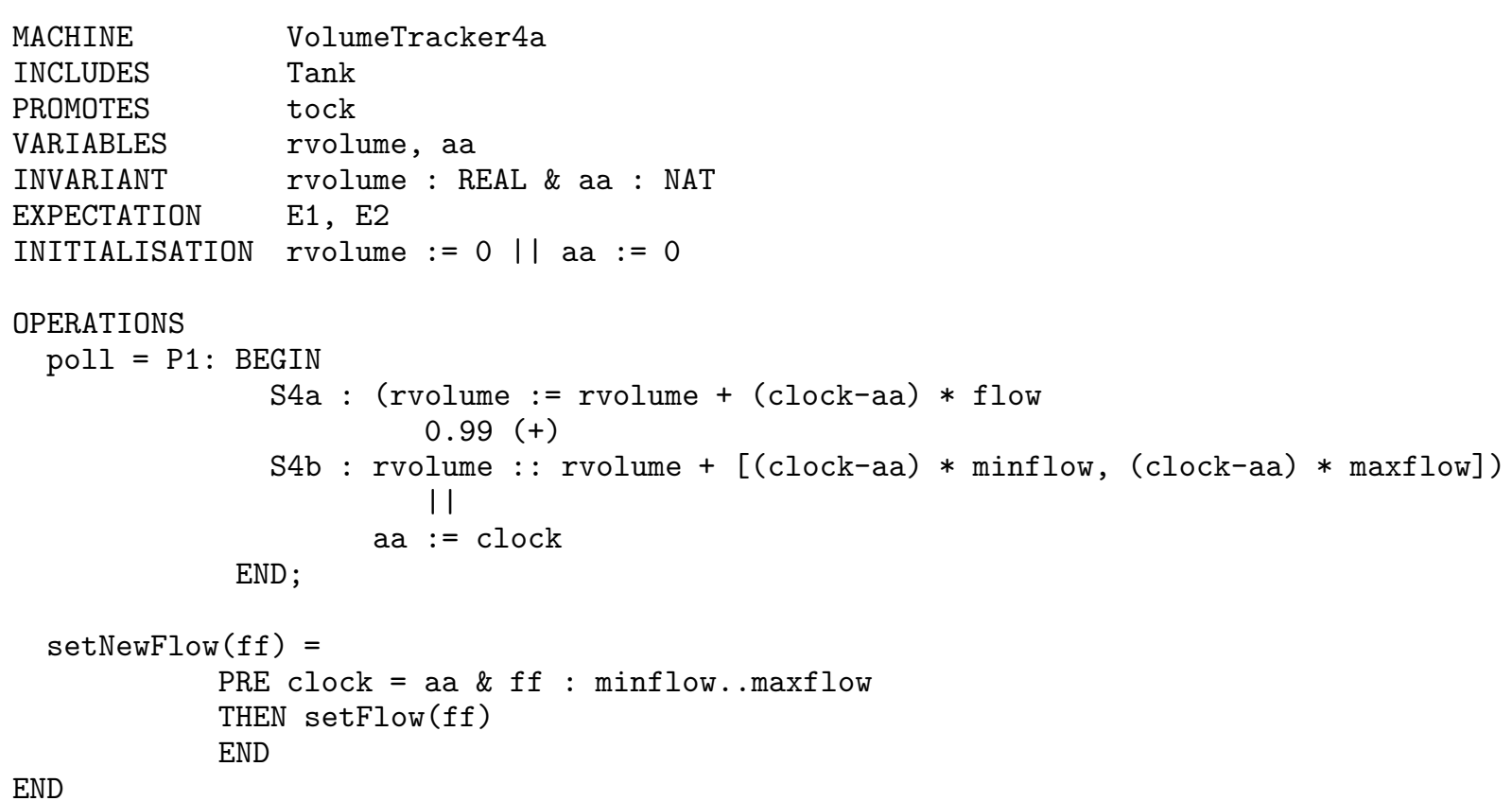

Fig. 16. The revised tank monitoring system incorporating explicit flow changes

\subsubsection{Summary}

The first approach to separating tock from poll yielded a very weak relationship between the system and the real values for the volume of the liquid in the tank, because assumptions about the way the operations would be executed were not built into the model. In this section we built in the assumption that tock and poll were dealing with the same values for flow by controlling more carefully when flow can be changed. We introduced a new operation setFlow to do this, but only when volume and rvolume were in step. We then required that the other operations could not alter flow. The expected operation of the system, whereby tock and poll will essentially occur together, is incorporated within this model. But an unexpected operation, in which tock and poll occurring together will read completely different values of flow, is not permitted within this mode of the system.

The result is a much tighter relationship on the expected values of volume and rvolume.

Note that the implementation of the poll operation in terms of sensors will again be the same as it was previously. All the changes we have made are at the level of the specification.

\section{Discussion}

The case study in this paper has shown how probabilistic B can be applied to specify and refine a system which naturally includes both probabilistic and nondeterministic behaviour, and has highlighted a number of issues that can arise in this process.

We considered two progressions of scenarios. The first progression was given in Section 5 . In the first scenario, we considered the simple case where sensor readings are either perfectly accurate, or completely arbitrary, with the sensors indicating whether they are working correctly or not. This enabled a value for the accuracy of the system's value rvolume to be given in terms of the range of possible flows. Essentially the accuracy is calculated by allowing for the worst case of nondeterminism, in accordance with the demonic approach to nondeterminism reflected in the semantics of the language. We obtained the expected result that the larger the ratio between the maximum and minimum flow, the less accurate the value we could expect.

In the second scenario, we allowed some error range on the values read even when the sensors were working correctly. This additional nondeterminism also entered into the calculation to determine the level 
of accuracy of rvolume, and again we saw that the wider the range of possibilities, for flow readings, and for the possible flows, the lower the level of accuracy for the system's record of the volume of liquid.

In the third scenario, the sensors no longer provided a direct indication of whether they were giving a correct reading or not, so it was necessary to use three sensors and compare readings to deduce which values are most likely correct. In this example we worked from the implementation to the specification, firstly obtaining the reliability provided by the combination of sensors, and then calculating the level of accuracy that the system could deliver.

All three of these scenarios were modelled using a machine which had only a single operation, which synchronised updates of the real tank and updates of the monitoring system.

In the second set of scenarios, we separated the model of the tank from the description of the monitoring system. This approach is more common in the development of embedded systems [DT97], and also occurs in Event-B [Abr96b, Abr03], since the separation allows a cleaner development of the system. The fact that different operations were used to update the states of the tank and of the monitoring system had a significant impact on the relationship between the expectations of the real volume and the monitoring system's value for it. We found that the first approach gave too weak a relationship, essentially no stronger than that provided by the invariant (which is concerned only with all possible reachable states). The reason for this is that probabilistic B does not provide any control on the invocation of machine operations, or assumptions on the order and frequency of their occurrence, so it must allow for the machine to be placed in any environment. The fact that the flow could change on any update of the tank meant that the system readings and the real flow values could be wildly different for some sequences of operation calls.

In the second scenario, we introduced behaviour incorporating realistic assumptions: that the flow would not change while the system updates and tank updates were out of step. We considered this reasonable because in practice these updates would tend to be in step. This assumption meant that the system readings for flow corresponded to the real flow into the tank, and we regained a tighter relationship between the expected values of the measured volume and the real volume of liquid.

We have seen that the requirement that every operation should not decrease the machine's expectation introduces a consistency condition between the expectation and the probabilistic and nondeterministic behaviour in the machine operations. This need for consistency can be pushed in either direction: either starting with a required expectation and then deriving the reliability requirements and flow parameters necessary to achieve that; or starting with a given combination of sensors with some known reliability and obtaining the tightest possible bounds on the expectation.

\section{Further work}

Although the case study was of a simple system, this paper has only explored some of the interesting kinds of behaviour that can arise in such systems, and many other scenarios remain ready to be explored. For example, we might wish to model sensors that take some time to be repaired once they break. Such modelling would most likely require some auxiliary variable to track the time left until the sensor is working correctly again, and the best way of modelling such a system in probabilistic B is far from clear.

Incorporating some information about the interactions between different operations raises some interesting problems. The final scenario we considered is quite relaxed in that it allows the measured volume to become quite out of step with the real volume. There are other possibilities for modelling such a scenario. For example, it might be preferable to introduce a stronger model of control flow to ensure that real updates and system updates occur alternately. This might require the introduction of flags to track which operation should be performed next, and guards to block operations from executing out of turn.

As an alternative, it may be appropriate to introduce controllers separately for probabilistic B machines, and combine them in the style of CSP $\| \mathrm{B}$ [TS00, TSB03]. Thus CSP processes will describe the permitted or expected sequences of operations, and could be used to drive the probabilistic B machine. This would allow some weaker requirements on expectations to be introduced in the context of such control loops: such expectations might need to be non-decreasing over the body of a control loop, rather than the stronger requirement that each operation individually should not decrease it. This is a topic for future research.

This work has concentrated on using probability with the abstract machine framework. More recent research has been investigating the use of probability in Event B [MTA05]. Event B [Abr96b, Abr03], again based on the B-Method, is a different framework which allows the specification to introduce more detail during the refinement steps. The refinement laws related to probability are not as mature as in this standard 
B framework and therefore it would be premature to compare the approach adopted in this paper with this very promising emerging work.

\subsection{Acknowledgements}

We are grateful to Neil Evans, Carroll Morgan, and Annabelle McIver for comments and discussions on this work. We are also grateful to the referees of this paper for their very thorough and detailed comments and suggestions which have led to improvements in the paper.

This research was initiated during Ken Robinson's and Thai Son Hoang's visit to Royal Holloway, University of London, in July 2003, and thanks are due to EPSRC for providing funds under grant GR96859/01 to support this visit.

\section{References}

[Abr96a] J-R. Abrial. The B-Book: Assigning Programs to Meanings. Cambridge University Press, 1996.

[Abr96b] J-R. Abrial. Extending B without changing it (for developing distributed systems). In 1st Conference on the B-Method, 1996.

[Abr03] Abrial. $\mathrm{b}^{\sharp}$ : Towards a synthesis between $\mathrm{Z}$ and B. In ZB2003: 3rd International Conference of $Z$ and B Users, number 2651 in LNCS. Springer, 2003.

[Dij76] E.W. Dijkstra. A Discipline of Programming. Prentice-Hall, 1976.

[DT97] J. Draper and H. Treharne. The refinement of embedded software with the B-Method. In Northern Formal Methods Workshop. Springer, 1997.

$\left[\mathrm{HJR}^{+}\right.$03] T.S. Hoang, Z. Jin, K. Robinson, A. McIver, and C. Morgan. Probabilistic invariants for probabilistic machines. In ZB2003: 3rd International Conference of $B$ and $Z$ Users, number 2651 in LNCS. Springer, 2003.

[MM04] A. McIver and C. Morgan. Abstraction, Refinement and Proof for Probabilistic Systems. Springer, 2004.

[MMH03] A. McIver, C. Morgan, and T.S. Hoang. Probabilistic termination in B. In ZB2003: 3rd International Conference of $B$ and $Z$ Users, number 2651 in LNCS. Springer, 2003.

[MMS96] C. Morgan, A. McIver, and K. Seidel. Probabilistic predicate transformers. ACM Transactions on Programming Languages and Systems, 18(3):325-353, 1996.

[Mor98] C. Morgan. The generalised substitution language extended to probabilistic programs. In B'98: the 2nd International B Conference, number 1393 in LNCS. Springer, 1998.

[MTA05] C.C. Morgan, Thai Son Hoang, and J-R. Abrial. The challenge of probabilistic event B. In ZB2005: 4th International Conference of $Z$ and $B$ Users, number 3455 in LNCS. Springer, 2005.

[Sch01] S. Schneider. The B-Method: an Introduction. Palgrave, 2001.

[STRT05] S. Schneider, Thai Son Hoang, K. Robinson, and H. Treharne. Tank monitoring: a pAMN case study. In REFINE'05, ENTCS, 2005.

[TS00] H. Treharne and S. Schneider. How to drive a B machine. In ZB2000: 1st International Conference of Z and B Users, number 1878 in LNCS. Springer, 2000.

[TSB03] H. Treharne, S. Schneider, and M. Bramble. Combining specification with composition. In ZB2003: 3rd International Conference of $Z$ and $B$ users, number 2651 in LNCS. Springer, 2003.

\section{A. Machine Readable pGSL}

This table gives the ascii form of statements in pGSL, used in the AMN descriptions presented in this paper. For a fuller account of machine-readable AMN, see [Abr96a, Sch01].

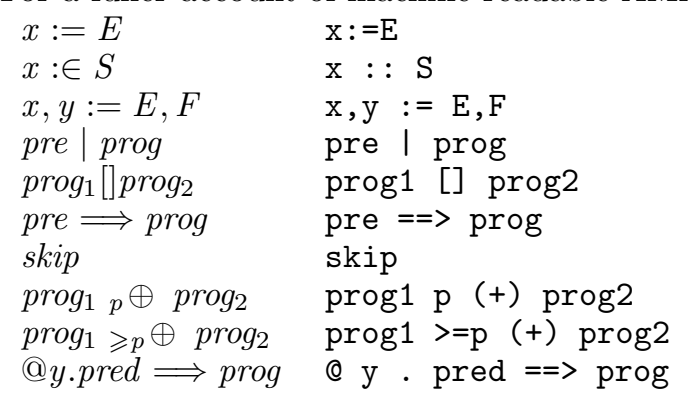




\section{B. Proof of Law A}

Law A states that:

$$
\operatorname{prog}_{2} \sqsubseteq \operatorname{prog}_{1} \Rightarrow \operatorname{prog}_{1} \geqslant p \oplus \operatorname{prog}_{2}=\operatorname{prog}_{1} \oplus \operatorname{prog}_{2}
$$

The proof makes use of some properties discussed in [MM04] Appendix B.1.1: idempotence of probabilistic choice, and monotonicity of pGSL operators with respect to the refinement ordering. It also makes use of the following quasi-associativity law given there as Law 3:

$$
\operatorname{prog}_{1 p} \oplus\left(\operatorname{prog}_{2} \oplus_{q / \bar{p}}\right)=\left(\operatorname{prog}_{1} p / \bar{q} \oplus \operatorname{prog}_{2}\right) \oplus_{q} \operatorname{prog}_{3}
$$

where $\oplus_{p}$ is the same as $\bar{p} \oplus$.

Firstly, we obtain immediately that

$\operatorname{prog}_{1 \geqslant p} \oplus \operatorname{prog}_{2} \sqsubseteq \operatorname{prog}_{1} \oplus \operatorname{prog}_{2}$

(Law 7 of [MM04] Appendix B.1.1)

Thus we have only to prove that

$$
\operatorname{prog}_{1} \oplus \operatorname{prog}_{2} \sqsubseteq \operatorname{prog}_{1 \geqslant p} \oplus \operatorname{prog}_{2}
$$

We first establish Lemma 1: If $\operatorname{prog}_{2} \sqsubseteq \operatorname{prog}_{1}$ and $p \leqslant q \leqslant 1$ then $\operatorname{prog}_{1} \oplus \operatorname{prog}_{2} \sqsubseteq \operatorname{prog}_{1} \oplus \operatorname{prog}_{2}$.

This is proved as follows:

$$
\begin{aligned}
& \operatorname{prog}_{1 p} \oplus \operatorname{prog}_{2} \\
& =\left\{\text { idempotence of }{ }_{p} \oplus, \bar{q} \leqslant \bar{p}\right\} \\
& \operatorname{prog}_{1 p} \oplus\left(\operatorname{prog}_{2} \oplus_{\bar{q} / \bar{p}} \quad \operatorname{prog}_{2}\right) \\
& \sqsubseteq\left\{\text { monotonicity, } \operatorname{prog}_{2} \sqsubseteq \operatorname{prog}_{1}\right\} \\
& \operatorname{prog}_{1 p} \oplus\left(\operatorname{prog}_{1} \oplus_{\bar{q} / \bar{p}} \quad \operatorname{prog}_{2}\right) \\
& =\{\text { quasi-associativity }\} \\
& \left(\operatorname{prog}_{1} p / q \oplus \operatorname{prog}_{1}\right) \oplus_{\bar{q}} \operatorname{prog}_{2} \\
& =\left\{\text { idempotence of }{ }_{p / q} \oplus\right\}
\end{aligned}
$$

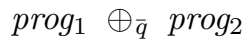

$$
\begin{aligned}
& =\left\{\oplus_{\bar{q}}={ }_{q} \oplus\right\} \\
& \operatorname{prog}_{1} \oplus \operatorname{prog}_{2}
\end{aligned}
$$

We are now in a position to establish line 4:

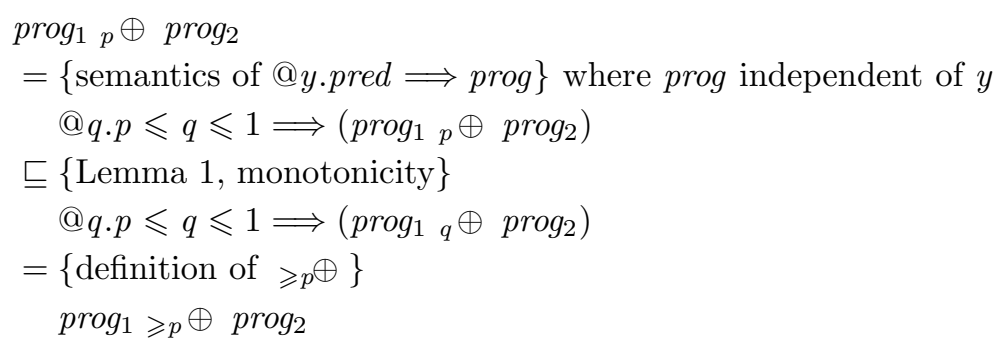

This concludes the proof.

\section{Calculation of expectation coefficients in VolumeTracker 2}

We calculate the requirement on $A$ from the requirement on $E 1$, as we did for VolumeTracker 1.

$$
\begin{aligned}
([\text { poll }] E 1)-E 1 & =([T \|(V 2 a 0.99 \oplus V 2 b)] E 1)-E 1 \\
& =([(T \| V 2 a) 0.99 \oplus(T \| V 2 b)] E 1)-E 1 \\
& =(0.99 \times[T \| V 2 a] E 1+0.01 \times[T \| V 2 b] E 1)-E 1
\end{aligned}
$$




$$
\begin{aligned}
& =(0.99 \times(\text { rvolume }+ \text { flow }+ \text { lowerror }-A \times(\text { volume }+ \text { flow })) \\
& +0.01 \times(\text { rvolume }+ \text { minflow }+ \text { lowerror }-A \times(\text { volume }+ \text { flow }))) \\
& -(\text { rvolume }-A \times \text { volume }) \\
& =0.99 \times(\text { flow }+ \text { lowerror }-A \times \text { flow })+0.01 \times(\text { minflow }+ \text { lowerror }-A \times \text { flow }) \\
& =(0.99-A) \times \text { flow }+0.01 \times \text { minflow }+ \text { lowerror }
\end{aligned}
$$

Observe in line (5) that the minimum expectation for the nondeterministic choice over [lowerror, higherror] occurs for the value lowerror.

Since ([poll $] E 1)-E 1$ must be non-negative for all values of flow, we obtain the requirement on $A$ that, for all values of flow, we have that

$$
A \leqslant 0.99+0.01 \times(\text { minflow } / \text { flow })+(\text { lowerror } / \text { flow })
$$

The expression takes its extreme values at flow $=$ minflow and flow $=$ maxflow, so we can obtain the constraints on $A$ by considering just these. Instantiating flow with minflow we obtain that

$$
A \leqslant 1+(\text { lowerror/minflow })
$$

and instantiating flow with maxflow we obtain that

$$
A \leqslant 0.99+0.01 \times(\text { minflow } / \text { maxflow })+(\text { lowerror } / \text { maxflow })
$$

Thus $A$ must be no greater than the minimum of these two values.

For $B$ we perform the following calculation:

$$
\begin{aligned}
([\text { poll }] E 2)-E 2= & \left(\left[T \|\left(V 2 a{ }_{0.99} \oplus V 2 b\right)\right] E 2\right)-E 2 \\
= & ([(T \| V 2 a) 0.99 \oplus(T \| V 2 b)] E 2)-E 2 \\
= & (0.99 \times[T \| V 2 a] E 2+0.01 \times[T \| V 2 b] E 2)-E 2 \\
= & (0.99 \times(B \times(\text { volume }+ \text { flow })-(\text { rvolume }+ \text { flow }+ \text { higherror })) \\
& \quad+0.01 \times(B \times(\text { volume }+ \text { flow })-(\text { rvolume }+ \text { maxflow }+ \text { higherror }))) \\
& \quad-(B \times \text { volume }- \text { rvolume }) \\
= & B \times \text { flow }-0.99 \times \text { flow }-0.01 \times \text { maxflow }- \text { higherror }
\end{aligned}
$$

We require that this is non-negative for any value of flow. Thus

$$
B \geqslant 0.99+0.01 \times(\text { maxflow } / \text { flow })+(\text { higherror } / \text { flow })
$$

for any value of flow. The expression takes its extreme values at flow $=$ minflow and flow $=$ maxflow, so we can obtain the constraints on $A$ by considering just these. Instantiating flow with minflow we obtain that

$$
B \geqslant 0.99+0.01 \times(\text { maxflow } / \text { minflow })+(\text { higherror } / \text { minflow })
$$

Instantiating flow with maxflow we obtain that

$$
B \geqslant 1+\text { (higherror/maxflow) }
$$

The first expression is greater than or equal to the second for any values of higherror, maxflow, minflow where minflow $\leqslant$ maxflow. Thus we obtain the condition on $B$ that

$$
B \geqslant 0.99+0.01 \times(\text { maxflow } / \text { minflow })+(\text { higherror } / \text { minflow })
$$

\section{Verifying the implementation of poll in VolumeTracker2I}

The poll operation in VolumeTracker $2 I$ is of the form $P 2 a ; P 2 b ; F ; R ; T$, where $v 1, v 2$, st 1 , st2, rflow are all local variables. We show that this operation is equivalent to poll given in the specification machine VolumeTracker2, as follows:

$P 2 a ; P 2 b ; F ; R ; T$

$=\{$ expanding $P 2 a$ and $P 2 b\}$ 


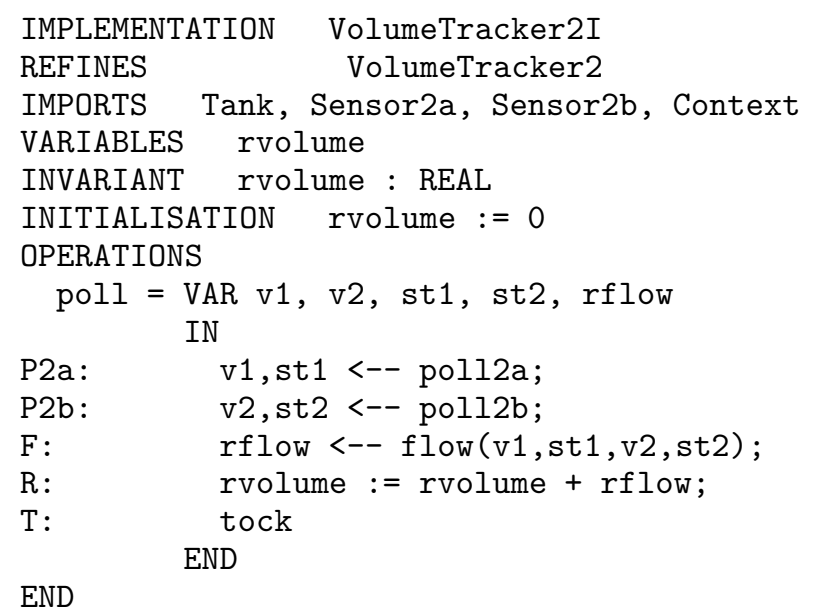

Fig. 17. The implementation VolumeTracker $2 I$

$(S 2 a l \geqslant 0.9 \oplus S 2 a r) ;(S 2 b l \geqslant 0.9 \oplus S 2 b r) ; F ; R ; T$

$=\{$ Law 15, then Law 20$\}$

$(S 2 a l ; S 2 b l ; F ; R ; T \geqslant 0.9 \oplus S 2 a l ; S 2 b r ; F ; R ; T)$

$\geqslant 0.9 \oplus$

$(S 2 a r ; S 2 b l ; F ; R ; T \geqslant 0.9 \oplus S 2 a r ; S 2 b r ; F ; R ; T)$

$=\{$ standard program algebra in each branch; removal of local variables $\}$

(rvolume :: rvolume + flow $+[(l e 2 a+l e 2 b) / 2,(h e 2 a+h e 2 b) / 2] ; T$

$\geqslant 0.9 \oplus$ rvolume $::$ rvolume + flow $+[$ le $2 a, h e 2 a] ; T)$

$\geqslant 0.9 \oplus$

(rvolume :: rvolume + flow $+[l e 2 b$, he $2 b] ; T$

$\geqslant 0.9 \oplus$ rvolume $::$ rvolume $+[$ minflow, $\operatorname{maxflow}] ; T)$

$\Leftarrow$ expanding the ranges of the nondeterministic choices,

provided lowerror $\leqslant l e 2 a$, lowerror $\leqslant l e 2 b$, he $2 a \leqslant$ higherror, he $2 b \leqslant$ higherror $\}$

(rvolume :: rvolume + flow $+[$ lowerror, higherror $] ; T$

$\geqslant 0.9 \oplus$ rvolume $::$ rvolume + flow $+[$ lowerror, higherror $] ; T)$

$\geqslant 0.9 \oplus$

(rvolume :: rvolume + flow $+[$ lowerror, higherror $] ; T$

$\geqslant 0.9 \oplus$ rvolume :: rvolume $+[$ minflow + lowerror, maxflow + higherror $] ; T)$

$=\{$ Laws 15 and 26$\}$

$V 2 a ; T \geqslant 0.99 \oplus V 2 b ; T$

$=\{$ Laws 15 and A, since $V 2 b \sqsubseteq V 2 a ; T$ independent of $V 2 a$ and $V 2 b\}$

$\left(V 2 a\left\|T_{0.99} \oplus V 2 b\right\| T\right)$

Thus we arrive at the operation poll given in the specification machine VolumeTracker2. This demonstrates that VolumeTracker $2 I$ indeed provides an implementation of VolumeTracker 2. 